\title{
Synchronization of Cubic Chaotic System
}

\author{
Yong Li (Corresponding author) \\ College of Science, Guilin University of Technology \\ Box 733, Guilin 541004, China \\ E-mail: gxbyl@163.com \\ Zhen Jia \\ College of Science, Guilin University of Technology \\ Box 733, Guilin 541004, China \\ E-mail: jjjzzz0@163.com
}

The research is financed by Guangxi Natural Science Foundation of China "multi-objective control and synchronization of Complex dynamic network" (No. 0991244) and Guangxi Education Department Research Projects of China "chaotic control and synchronization of complex dynamic network"(No. 200807MS043)

\begin{abstract}
This paper utilizes an adaptive synchronization control principle based on OGY idea to realize the synchronous of two cubic chaotic systems which have different initial value and parameter. Then theory and numerical simulation shows that the control strategy needs to select right error Regions' Radius $\delta$ and feedback coefficient $\omega$. The paper also utilizes control principle of lag synchronization to realize the lag synchronization of cubic chaotic system and Logistic map. Then theory and numerical simulation shows that state variables of Logistic map track state variables of cubic chaotic system strictly.
\end{abstract}

Keywords: Cubic chaotic system, Logistic map, Adaptive synchronization, Lag synchronization

\section{Introduction}

Since the pioneering work on chaotic control was introduced by Ott, Grebogi and Yorke (OGY) (E. Ott, C. Grebogi, \& J. A. Yorke, 1990, p.1196), chaos control has gained wide and intensive research. Then, in 1990, Pecora and Carroll proposed drive-response synchronization proposal. Furthermore, synchronization of chaos and its potential applications in secure communication have caused widespread concern since drive-response synchronization has been realized in the electronic circuit firstly (L. M. Pecora , \& T. L. Carroll, 1990, p.821) . Over the decades, many methods and techniques for chaos control and synchronization had been produced, such as OGY method (E. Ott, C. Grebogi, \& J. A. Yorke, 1990, p.1196), PC method (L. M. Pecora , \& T. L. Carroll, 1990, p.821), feedback approach (Y .Wang, Z. Guan, \& H. O. Wang, 2003, p.34), adaptive method (Y .Wang, Z. Guan, \& H. O. Wang, 2003, p.34), time-delay feedback approach(G. Chen, \& X. Dong, 1998), backstepping design technique (X. Wu, \& J. Lu, 2003, p.721 ), etc. Chaos control is increasingly forming a systematic theoretical system.

Generally, nonlinear dynamic systems can be divided into two major categories of continuous systems and discrete systems. Yet according to Poincare section method, n-dimensional continuous system can change into (n-1)-dimensional discrete systems, so the study of the control of discrete chaotic systems has universal significance. The Lyapunov design for nonlinear discrete-time systems becomes much more intractable than in the continuous-time. The reason lies in that the linearity property of the derivative of a Lyapunov function in continuous-time is not present in the difference of Lyapunov function in the discrete-time (Y. Song, \& J. W. Grizzle, 1993, pp.1359-1364). Many controls designed for continuous-time systems may be not suitable for discrete-time systems due to some inherent difficulties in discrete-time models.

This paper will study adaptive synchronization of two cubic chaotic systems with same structure and lag synchronization of cubic chaotic systems and Logistic map with different structure. The dynamics properties of cubic chaos have been discussed in literature (Li Yong, Jia Zhen, etc, 2010, 5(2)) in detail. Cubic Chaos can be expressed as differential equations:

$$
x_{n+1}=\lambda x_{n}-x_{n}{ }^{3} \quad \lambda \in(0,3)
$$


Initial value of chaos $x_{0} \in(-2,2)$ and threshold $\lambda_{\infty}=2.3489175 \cdots \cdots$ While $\lambda \in\left(\lambda_{\infty}, 3\right)$, system enter the chaotic zone. Yet most periodic solution of Lorenz system is in accordance with one-dimensional symmetric periodic orbits of cubic mapping order and the iterative as follows(C. Grebogi, \& J.A.Yorke, 2001, pp.135-136): $x_{n+1}=\lambda x_{n}+(1-\lambda) x_{n}^{3}$

Clearly, while $\lambda=2$, the antisymmetric cubic map is cubic chaos. The cubic chaos system has more complex dynamics properties. Therefore, cubic chaotic system (1) has significance to periodic solution of Lorenz system.

Logistic map (Hassan K. Khalil, 2005)can be expressed as differential equations:

$$
z_{n+1}=\mu z_{n}\left(1-z_{n}\right) \quad \mu \in(0,4)
$$

Because of dependent sensitivity on initial values and its ergodicity of chaotic track, as well as based on OGY idea, this paper designs an adaptive synchronization control strategy. It fully shows the OGY method is able to make minor adjustments on the parameters. Theory and numerical simulation shows that the control strategy selects the appropriate region radius and the error feedback coefficients can achieve faster adaptive synchronization of two cubic chaos systems. What's more, because of the controller of the response parameters is divided into three kinds, yet the pre-control is not a member of controlled substance, so this design is different from continuous adaptive control strategy (Wang Jie, \& Zhang Huaguang, 1999, pp.217-221, He Mingfeng, MuYunming, \& Zhao Lizhong, 2000, pp.830-832) which has joined the controller of each iteration and synchronous control strategy (Dai Dong, \& Ma Xikui, 2001, pp.1237-1240) which has joined the controller intermittently. This paper adds in controller when errors of the two tracks reach a certain neighborhood. In the practical application of information engineering, electronic technology and other field, the less control costs means that the greater the economic effects and practical value. Based on lag synchronization control principl (Hu Gang, Xiao Jinghua, \& Zheng Zhigang, 2000, pp78-85), for different structures, we select typical one-dimensional mapping----Logistic map as a response system. This lag synchronization controller has a certain universal, and have some reference value for the other lag synchronization of different structures on studying.

\section{Synchronization with the same structure for Cubic chaotic systems}

\subsection{Adaptive synchronization control principle based on OGY idea}

Consider two same structure of discrete chaotic systems in the following form:

$$
\begin{aligned}
& x_{n+1}=f\left(x_{n}, \lambda_{c}\right) \\
& y_{n+1}=f\left(y_{n}, \lambda_{n}\right)
\end{aligned}
$$

where $n \in Z^{+}, x_{n}, y_{n}$ are system states, $x, y \in R^{N} . \lambda_{c}$ is system parameter and $\lambda_{n}$ is variable adjustable parameter, $f: R^{N} \rightarrow R^{N}$. If we lead control law $\lambda_{n+1}=G\left(\lambda_{n}\right)$ into adjustable parameter $\lambda_{n}$ of the response system, and satisfies $\lim _{t \rightarrow+\infty}\left|y_{n}-x_{n}\right|=0$, we will have system (3) and (4) achieving adaptive synchronization.

Next by controlling the input data of response system and system parameters, the adaptive synchronization method of two cubic chaotic systems are given as follows:

$$
\begin{aligned}
& x_{n+1}=\lambda_{c} x_{n}-x_{n}^{3} \\
& y_{n+1}=\lambda_{n} x_{n}-x_{n}^{3} \\
& \lambda_{n+1}=\lambda_{n}+u_{n}
\end{aligned}
$$

where $\lambda_{c}, \lambda_{n} \in(2.35,3), x_{0}, y_{0} \in(-2,2)$. Regarding arbitrary initial value $x_{0}, y_{0}$, their tracks are irrelevant.

The adaptive synchronization error system is given as follows:

$$
e_{n+1}=y_{n+1}-x_{n+1}
$$

The adaptive synchronization of two cubic chaotic systems transforms to control the error $e_{n+1}$ on $\lim _{n \rightarrow+\infty} e_{n+1}=0$, that is realize precise synchronization of cubic chaotic system (5) and (6).

Because chaotic systems evolution sensitively depends on the initial conditions, if parameters and initial value of 
cubic chaotic systems are not the same, their orbits are disorganized and irrelevant. But the dynamics in the chaotic attractor is ergodic, so the orbits of two systems maybe close to each other even intersect, coincide in the course of evolution. However, after a period of time, they will automatically stay away from each other for reasons such as disturbances. Based on OGY method, while the radius of error system less than $\delta$, we can add controller to the systems to make the two systems reach synchronization after a period of time sequence.

Thus, we design adaptive parameter synchronization control law as Theorem 1.

Theorem 1. Regarding system (5) and (6), if controller as follows is added into iterative equations on the parameters (7),

$$
\begin{aligned}
\text { If } \lambda_{0}<\lambda_{c}, u_{n}= \begin{cases}0 & e_{n+1} \in(-\infty,-\delta] \cup(\delta,+\infty) \\
\omega e_{n+1} & e_{n+1} \in(0, \delta] \\
-\omega e_{n+1} & e_{n+1} \in(-\delta, 0]\end{cases} \\
\text { If } \lambda_{0}>\lambda_{c}, u_{n}= \begin{cases}0 & e_{n+1} \in(-\infty,-\delta] \cup(\delta,+\infty) \\
\omega e_{n+1} & e_{n+1} \in(-\delta, 0] \\
-\omega e_{n+1} & e_{n+1} \in(0, \delta]\end{cases}
\end{aligned}
$$

where $\delta>0$ is a enough small error regions' radius, $\omega \in(0,1)$ is feedback coefficient, system (5) and (6) will achieve adaptive synchronization.

Proof. To prove briefly, we suppose the controller $u_{n}=\varepsilon e_{n+1}, \varepsilon$ is feedback coefficient. We mark $\Delta \lambda_{n}=\lambda_{n}-\lambda_{c}$. From (5) and (6), we can get

$$
e_{n+1}=\left(\lambda_{n}-\lambda_{c}\right) x_{n}=\Delta \lambda_{n} x_{n}
$$

Bring (9) into (7), we get

$$
\lambda_{n+1}=\lambda_{n}+\varepsilon \Delta \lambda_{n} x_{n}
$$

Subtract $\lambda_{c}$ on both sides, we get

$$
\Delta \lambda_{n+1}=\Delta \lambda_{n}+\varepsilon \Delta \lambda_{n} x_{n}=\Delta \lambda_{n}\left(1+\varepsilon x_{n}\right)
$$

That is

$$
\Delta \lambda_{n+1}=\Delta \lambda_{0} \prod_{i=1}^{n}\left(1+\varepsilon x_{i}\right)
$$

If $\left|1+\varepsilon x_{i}\right|<1$, it must be $\lim _{n \rightarrow+\infty} \prod_{i=1}^{n}\left(1+\varepsilon x_{i}\right)=0$, then $\lim _{n \rightarrow+\infty} \Delta \lambda_{n}=0$. Due to (9), we can see $\lim _{n \rightarrow+\infty} e_{n}=0$. Because $x_{0} \in(-2,2)$, we will get $x_{i} \in(-2,2)$. Two cases will be discussed below. While $x_{i} \in(-2,0)$, $\varepsilon \in(0,1)$; while $x_{i} \in(0,2), \varepsilon \in(-1,0)$. In these cases, $\left|1+\varepsilon x_{i}\right|<1, \lim _{n \rightarrow+\infty} e_{n}=0$.

Then we will study the relationship of feedback coefficient $\varepsilon$, error system $e_{n+1}$ and $\Delta \lambda_{n}$. From (9), we can see that symbol of error system $e_{n+1}$ is decided by product of $x_{n}$ and $\Delta \lambda_{n}$, yet symbol of $x_{n}$ determines symbol of feedback coefficient $\varepsilon$. From (9), we can get $x_{n}=e_{n+1} /\left(\lambda_{n}-\lambda_{c}\right)$. We give a proper radius of the error region $\delta>0$, then we will find that while $x_{i} \in(0,2)$ and $\lambda_{n}>\lambda_{c}, e_{n+1} \in(0, \delta)$; while $x_{i} \in(0,2)$ and $\lambda_{n}<\lambda_{c}, e_{n+1} \in(-\delta, 0)$, and now the feedback coefficient $\varepsilon \in(-1,0)$. While $x_{i} \in(-2,0)$ and $\lambda_{n}<\lambda_{c}, e_{n+1} \in(0, \delta)$; while $x_{i} \in(-2,0)$ and $\lambda_{n}>\lambda_{c}, e_{n+1} \in(-\delta, 0)$, and now the feedback coefficient $\varepsilon \in(0,1)$. Yet $\lambda_{n}$ converges $\lambda_{c}$ uniformly, so symbol of $\Delta \lambda_{n}$ is determined by $\lambda_{0}$ 
and $\lambda_{c}$. Thus we get theorem 1 .

Remark 1. Essentially, as cubic chaotic systems, while the absolute value of the error trajectory less than $\delta$, we add in the control law (8). In fact, the synchronous control law made three kinds of different control strategies based on specific circumstances. The first which is called pre-control $\left(u_{R}=0\right)$ don't add in controller when trajectory error is larger; the second which is called positive feedback control $\left(u_{P}=\omega e_{n}\right)$ aim to pre-reduce the negative feedback control error in the next step; the third which is called negative feedback control $\left(u_{N}=-\omega e_{n}\right)$ aim to reduce the error of the two systems track.

\subsection{Numerical simulation and Conclusion}

Select parameter $\lambda_{c}=2.9$ of the drive system (5) and initial parameter $\lambda_{0}=2.7$ of response system (6). We assume that initial value is $x_{0}=0.45, y_{0}=0.5$, error regions' radius is $\delta=0.1$, feedback coefficient is $\omega=0.5$, iteration steps is $n=100$. Adaptive synchronization error figure of the system (5) and (6) as shown in figure 1, we can see the two systems reach adaptive precise synchronization after about 30 iteration steps. In the 100's iteration process of the system, pre-control, positive feedback control and negative feedback control occur 5 times, 17 times and 78 times respectively. Each iteration by adding the control as shown in figure 2. Evidently, after about 10 iteration steps, the parameter of response system only carries out positive feedback control and negative feedback control. This shows that after adding in controller, the track of the two systems will be closer and reach synchronization finally. Convergence curve of parameter $\lambda_{n}$ of response system as shown in figure 3 . We can see parameter $\lambda_{n}$ of response system converge to the parameter $\lambda_{c}=2.9$ of drive system gradually until reaching adaptive synchronization completely. As shown in figure 4, synchronization signal of two cubic chaotic systems is expressed with Lissajous figure.

Remark 2. (1) If error regions' radius $\delta$ is too large, the parameter $\lambda_{n}>3$ maybe occur after a few iterative steps but the system doesn't achieve synchronization. If $\delta$ is too small, it will be too difficult to add in satisfied positive and negative feedback control. In other words, the probability of adding in effective control will be lower and the system needs a lot of iterations to achieve synchronization. What's worse, the error system will be led to divergent gradually and impossible to achieve adaptive synchronization finally.

(2) If feedback coefficient $\omega$ is too large, magnitude of the changes of parameter is larger and leads to synchronization faster. If $\omega$ is too small, the rate of the synchronization will be too slow to have practical significance.

\section{Synchronization of cubic chaotic system and Logistic map of different structure}

\subsection{Control principles of lag synchronization}

Regarding the system (10) that is composed of two discrete chaotic dynamical systems,

$$
\left\{\begin{array}{l}
x_{n+1}=f\left(x_{n}, p\right) \\
y_{n+1}=g\left(y_{n}, q\right)
\end{array}\right.
$$

where $x_{n}, y_{n} \in R^{N}, n, N \in Z^{+}$are system states, $p, q$ are system parameters, $f, g: R^{N} \rightarrow R^{N}$, and there is a constant $\tau$ independent of time but satisfy $\lim _{t \rightarrow+\infty}\|y(t)-x(t-\tau)\|=0$, system (10) will achieve lag synchronization.

We will study lag synchronization of two discrete systems of different structure as follows. We make the system (11) as a drive system and the system (12) as a controlled response system.

$$
\begin{aligned}
& x_{n+1}=A x_{n}+F\left(x_{n}\right) \\
& y_{n+1}=B y_{n}+G\left(y_{n}\right)+u_{n}
\end{aligned}
$$

where $n, N \in Z^{+}, x \in R^{N}, y \in R^{N}, A \in R^{N \times N}, B \in R^{N \times N} . u_{n} \in R^{N}$ is lag Synchronization controller. $F\left(x_{n}\right), G\left(y_{n}\right)$ is the nonlinear function.

Error system is 
$e_{n}=y_{n}-x_{n-\tau}$

Then

$$
e_{n+1}=y_{n+1}-x_{n+1-\tau}=B y_{n}-A x_{n-\tau}+G\left(y_{n}\right)-F\left(x_{n-\tau}\right)+u_{n}
$$

Select

$$
u_{n}=S\left(x_{n-\tau}\right)-T\left(y_{n}\right)
$$

where

$$
S\left(x_{n}\right)=F\left(x_{n}\right)+(A-B+K) x_{n}, T\left(y_{n}\right)=G\left(y_{n}\right)+K y_{n}, K \in R^{N \times N}
$$

From (13) and (14), we get

$$
e_{n+1}=(B-K) y_{n}-(B-K) x_{n-\tau}=(B-K) e_{n}=C e_{n}
$$

Coefficient matrix of error system (15) is $C \in R^{N \times N}$, and its eigenvalue equation is: $|\lambda I-C|=0$. According to system stability theory and by pole placement technique, we select appropriate debugging matrix $K$ to satisfy that the characteristic values of $C=B-K$ has negative real parts, thereby $\lim _{n \rightarrow+\infty} e_{n}=0$. Then drive system and response system will achieve synchronization.

From the above discussion, for the cubic system (1) and the Logistic map (2), we can get the following theorem.

Theorem 2. For the driving response system (16) which is constituted of the cubic chaotic system (1) and the controlled response Logistic map (2),

$$
\left\{\begin{array}{l}
x_{n+1}=\lambda x_{n}-x_{n}^{3} \\
z_{n+1}=\mu z_{n}\left(1-z_{n}\right)+u_{n}
\end{array},\right.
$$

if lag synchronization controller $u_{n}=S\left(x_{n-\tau}\right)-T\left(z_{n}\right)$, where

$$
\left\{\begin{array}{l}
S\left(x_{n}\right)=-x_{n}^{3}+(\lambda-\mu+k) x_{n} \\
T\left(z_{n}\right)=-\mu z_{n}^{2}+k z_{n}
\end{array}\right.
$$

and if $k>\mu>0$, system (16) will achieve lag synchronization.

\subsection{Numerical simulation and Conclusion}

Firstly, we give bifurcation diagram of cubic chaotic system (1) and Logistic map (2). They are shown in figure 5(a)(b) respectively. From the diagram, we can find the two systems have different dynamic behaviors.

Then, we use MATLAB and make numerical simulations according to the control strategy described in Theorem 2. Select initial value $x_{0}=0.3, z_{0}=-0.1$ and system parameters $\lambda=2.9, \mu=3.8$, we will find that the two systems are in a chaotic state and retardation $\tau=10$, debug parameter $k=4.2$. Error of the two systems changes over time and we can see the process of evolution on figure 6 . After 18 iterative steps, error of the two systems approach zero gradually, as well as drive and response system reach a deferred synchronous quickly. If the system parameters and debug parameter take other reasonable values, we can get the desired results similarly though there are some difference on the rate of synchronous. State variables of the two systems change over time and we can see the process of evolution on figure 7. State variable $z_{n}$ of Logistic map tracks state variable $x_{n}$ of cubic chaotic system strictly. This shows that the controller is feasible.

\section{Conclusion}

For chaotic systems is sensitive to the initial value, even if the parameters of the chaotic system of two identical initial difference is small, their trajectories will diverge exponentially with time. In the actual application, from the noise and interference, and system hardware perspective, it is difficult to find two systems with exactly the same parameters. Therefore, the application of the synchronization of two different parameters of chaotic systems is important in practical. This paper utilizes an adaptive synchronization control principle based on OGY 
idea to realize the adaptive synchronization of two cubic chaotic systems which have same structure but different initial value and parameter. Moreover, the paper also utilizes control principle of lag synchronization to realize the lag synchronization of cubic chaotic system and Logistic map, and here the two are discrete system of different structure. Theory and numerical simulation shows the effectiveness and practicality of the two control strategies.

\section{References}

C. Grebogi, \& J.A.Yorke.(2001). The Impact of Chaos on Science and Society. Yang Li, \& Liu Jubin, etc. translation. Hunan Science and Technology Press. pp.135 -136.

Dai Dong, \& Ma Xikui. (2001). Chaos synchronization on using intermittent parametric adaptive control. Acta Physica Sinica. 50(7). 1237-1240.

E. Ott, C. Grebogi, \& J. A. Yorke. (1990) Phys. Rev. Lett. 64 p.1196.

G. Chen, \& X. Dong(1998), From Chaos to Order: Perspectives, Methodologies and Applications. World Scientific, Singapore.

Hassan K. Khalil. (2005). Nonlinear Systems (3rd ed.). Bei Jing: Publishing House of Electronics Industry .

He Mingfeng, MuYunming, \& Zhao Lizhong. (2000). Synchronization on using parametric adaptive control algorithm. Acta Physica Sinica. 49(5).pp. 830-832.

$\mathrm{Hu}$ Gang, Xiao Jinghua, \& Zheng Zhigang. (2000). Chaos Control. Shang Hai: Shang hai Scientific and Technological Education Publishing House. pp.78-85.

L. M. Pecora , \& T. L. Carroll(1990). Phys. Rev. Lett. 64. p.821.

Li Guohui, Xu Deming, \& Zhou Shiping.(2004). Chaos synchronization by using random parametric adaptive control method . Acta Physica Sinica. 53(2).pp.379-382.

Li Yong, Jia Zhen, Wang Jun, \& Wang He. (2010). The dynamics properties of a discrete chaotic system . Journal of GuiLin University of Technology. 5(2). China.

Wang Jie, \& Zhang Huaguang. (1999) Parametric Adaptive control of discrete chaotic systems. Control and Decision.14(3).pp. 217-221.

X. Wu, \& J. Lu, (2003). Chaos Solitons Fractals 18. p.721.

Y. Song, \& J. W. Grizzle,(1993) Adaptive output-feedback control of a class of discrete-time nonlinear systems. Proceedings of American Control Conference. pp. 1359-1364.

Y .Wang, Z. Guan, \& H. O. Wang (2003). Phys. Lett. A 312 p. 34.

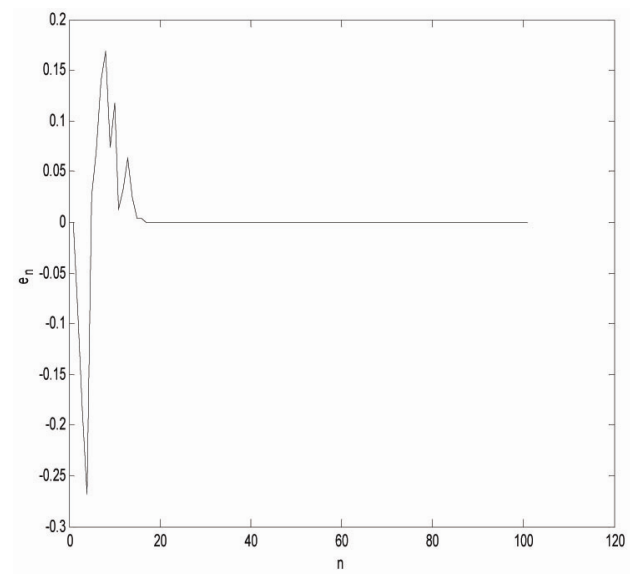

Figure 1. Synchronization error of system (5) and (6)

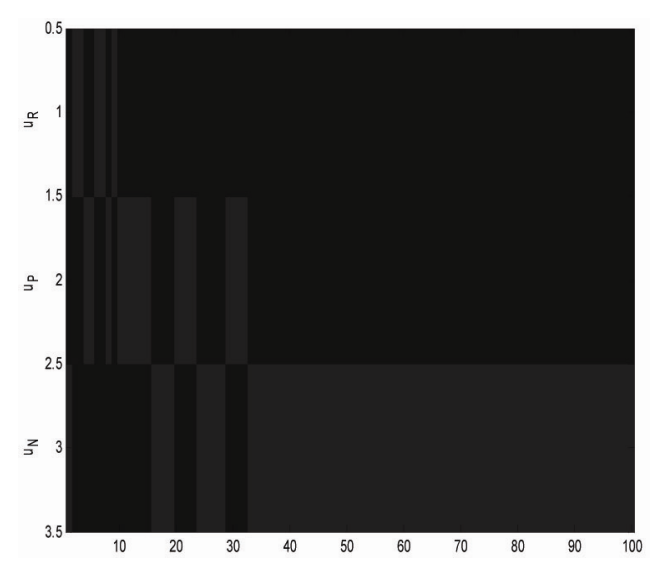

Figure 2. Actual figure of controller (8) 


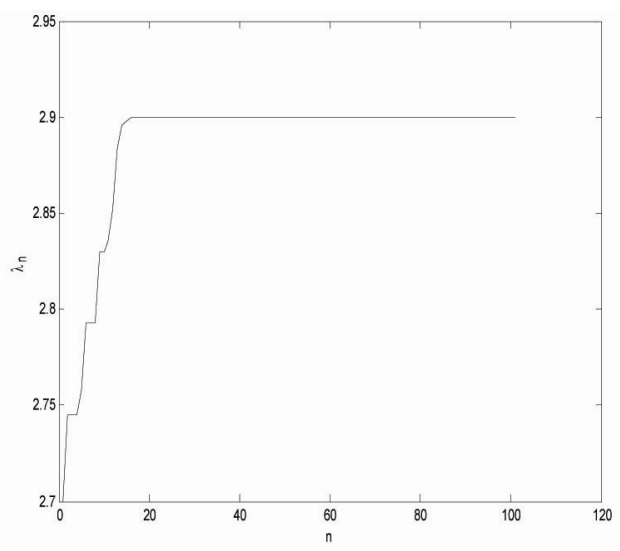

Figure 3. Convergence curve of $\lambda_{n}$

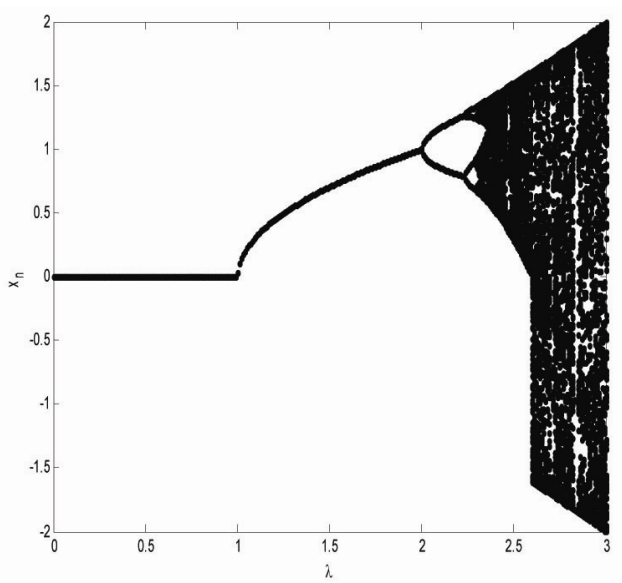

(a)

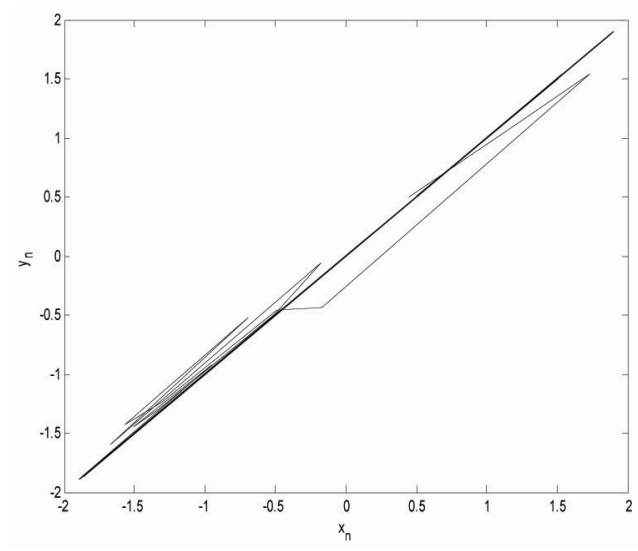

Figure 4. Lissajous figure of system (5) and (6)



(b)

Figure 5. Bifurcation diagram of cubic chaotic system(1) and Logistic map (2)

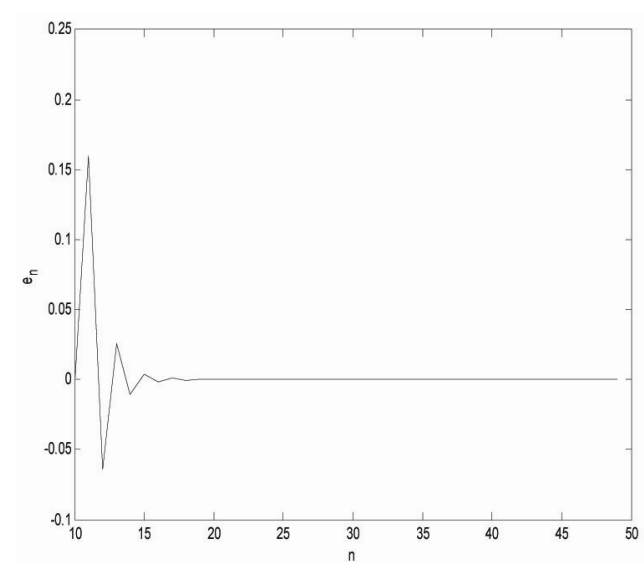

Figure 6. Synchronization error of system(16)

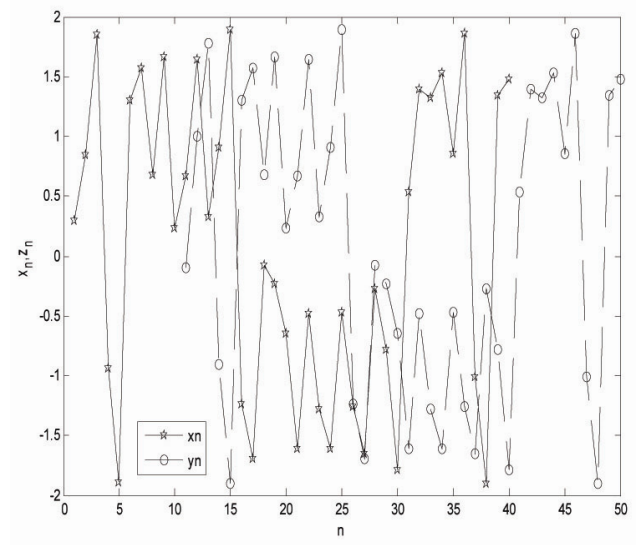

Figure 7. Synchronization process of state variable 